\title{
Online state and input force estimation for multibody models employing extended Kalman filtering
}

\author{
Frank Naets · Roland Pastorino · Javier \\ Cuadrado - Wim Desmet
}

Received: date / Accepted: date

\begin{abstract}
This paper discusses the use of Sub-System Global Modal Parameterization (SSGMP) reduced multibody models in an augmented discrete extended Kalman filter (ADEKF) to generate a general formalism for online coupled state/input estimation in mechanisms. The SS-GMP approach is proposed to reduce a general multibody model of a mechanical system into a real-time capable model without considerable loss in accuracy. In order to use these reduced models with an extended Kalman filter, the necessary derivatives of this model are provided. An exponential integration scheme is used to discretize the model in order to be compatible with discrete time filters. Finally, the augmented approach is used for the estimation, in which the unknown external forces are considered as additional states to be estimated. The proposed approach is validated numerically and compared to three other filtering approaches. The validation demonstrates that the proposed approach provides accurate results while still maintaining real-time performance.
\end{abstract}

Keywords Global Modal Parameterization · Kalman filter · force estimation

\section{Introduction}

In many mechanical applications, there is a large need to know the current state of the system: for control purposes it is crucial to take appropriate action, for health monitoring the condition of the system has to be known, ... The most straightforward approach to this problem is the direct measurement of the state of interest. Quite often however, this is not feasible. Measurement location can be hard to reach, sensors can be prohibitively expensive or no sensor might even exist for the variable of interest. In order to address this issue, state estimators have made a huge rise over the last decades. This rise was kickstarted by the introduction of the Kalman filter [1], and many variations of this approach have been presented over the years [2]. The main strength of this method is that it optimally leverages the a-priori knowledge of a system, by the use of a model, and the measurements.

Frank Naets

Department of Mechanical Engineeringm, Katholieke Universiteit Leuvenm, Celestijnenlaan 300B, B-3001 Heverlee

E-mail: frank.naets@mech.kuleuven.be 
For many mechanical systems however, there are two main practical problems in stateestimation:

- design of an appropriate system nominal model,

- unknown input forces.

The state-estimator generally requires an accurate model in order to provide good results. Currently the general approach is still to create (simplified) ad-hoc models for the use in the filter [3-5]. The trade-off which has to be kept in mind when creating these models is between computational load and accuracy. This process of specific model generation is typically time-consuming and it can be difficult to tune the parameters of these models to match the real system. Multibody simulation provides a general framework to develop high fidelity models for mechanical systems [6]. Ideally these models would also be applied for the state-estimation of these systems [7,8]. Unfortunately this last approach leads to models which cannot be run in real-time together with an extended Kalman filter for systems with multiple degrees-of-freedom (DOFs). In order to allow the use of complex design models for state-estimation purposes, this work proposes a nonlinear model reduction technique for multibody systems, namely the Sub-System Global Modal Parameterization (SS-GMP). This method is a system-level model reduction technique for multibody systems. Whereas the original GMP approach $[9,10]$ did not perform well in the case of multiple DOFs, SSGMP allows the division of a mechanism into multiple sub-models which can be efficiently reduced by GMP and can then be connected back together [11]. In this work the SS-GMP approach is proposed for systems consisting of rigid bodies and localized force elements. A brief summary of the methodology is provided in Sec. 2. Special attention is given to the formulation of the appropriate derivative matrices required for the evaluation of the Kalman filter. In practice, also the determination of the parameters of the model might be an issue. These parameters could be obtained through a proper parameter identification beforehand or could also be updated through parameter estimation techniques [12-14], which show similarities to the methods proposed in this work.

The second issue for many mechanical systems is the fact that the input-forces are not known. Regular state estimators assume that the inputs to the system are known, but in practice this is rarely the case. This typically leads to bias-errors on the state estimates and degraded performance. In order to alleviate this issue, several authors have proposed biasfree Kalman filters $[15,16]$. However, in mechanical systems it is often of special interest to obtain an estimate of the external loads to a system as well. This is clearly the case in vehicle applications where the unknown friction coefficient $[17,18]$ and vertical displacement of the road is usually unknown but crucial for proper control. However this issue can be extended to many other domains as well, like mechatronics [19-21] and biomechanics. Several variations of the regular Kalman filter have been proposed to perform combined state and input estimation [22-24]. These approaches can be categorized under unknown input observers [25] and have the advantage that they provide a simultaneous estimation of states and forces. Even though accelerometers are commonly used in mechanical applications, cheap gyroscopic sensors based on MEMS technology are becoming increasingly popular. Therefore it is likely that no direct feedthrough from the inputs to the measurements is present and the approach by Gillijns et al. [24] is not applicable. In this work the augmented Kalman filter is chosen in order to take the unknown inputs into account [22], which has been applied with success to linear [26] and nonlinear [17] mechanical systems. In this work the augmented approach is applied to an extended Kalman filter due to the nonlinear equations of motion for the multibody system. Moreover, in order to maximize real-time performance the filter is described in the discrete time domain, as formulation is more suit- 
able for efficient implementation. The resultant filtering approach is an augmented discrete extended Kalman filter (A-DEKF). The algorithm for this filter is discussed in Sec. 3. In order to obtain discrete time equations from the continuous time equations of motion for the multibody system, an exponential discretization [27] is applied.

Finally the proposed approach is validated numerically in Sec. 4. A planar half-car system is simulated with realistic measurement properties. The proposed approach is shown to provide superior performance to two basic disturbance estimators and a linearized A-DKF. Moreover the proposed A-DEKF approach coupled with an SS-GMP model is shown to be real-time capable through an implementation in Fortran.

\section{Connected Sub-System Global Modal Parameterization}

In this work the Sub-System Global Modal Parameterization (SS-GMP) approach is proposed as model reduction method to create models for mechanical systems for use in state estimators. This approach was first introduced in the frame of real-time simulation of flexible multibody systems [11] and is applied to rigid mechanisms in this work.

In this section, equations are presented for the planar case for the sake of clarity, but results can be easily extended to the spatial case as presented by Naets [11] for flexible systems.

The SS-GMP modeling approach consists of two steps [11]:

- Preprocessing: during this phase, the model is split up into sub-models which can be reduced separately. Subsequently the reduction, according to the GMP approach $[9,10]$, is performed for a grid of possible configurations and the system matrices are stored for each sub-model.

- Processing: during the simulation, the equations of motion for each sub-model are evaluated based on the interpolation of the stored system matrices and the equations presented hereafter. After each sub-model is evaluated, the redundant DOFs are eliminated and the equations of motion for the full system are evaluated.

\subsection{Dividing model into sub-models}

The SS-GMP is developed specifically for mechanical systems which are the assembly of multiple sub-mechanisms with closed kinematic loops (Fig. 1). In the case of a regular GMP reduction, systems with a large number of independent DOFs would lead to a very highdimensional reduced model which could not be run efficiently. The SS-GMP approach on the other hand is based on the division of a complex system into smaller systems with unconnected independent DOFs except for a common mechanism attached frame (MAF), as shown in Fig. 1.

Once the model of the full system is split into sub-models, these sub-models are reduced according to the GMP approach such that their behavior can be represented by a reduced unconstrained formulation with a minimal number of DOFs.

Irrespective of the original model formulation (Cartesian coordinates, natural coordinates, ... ) the reduced generalized coordinates $q$ for one sub-model $i$ are:

$$
q_{i}=\left[\begin{array}{c}
x_{0} \\
p_{0} \\
\theta_{i}
\end{array}\right] .
$$




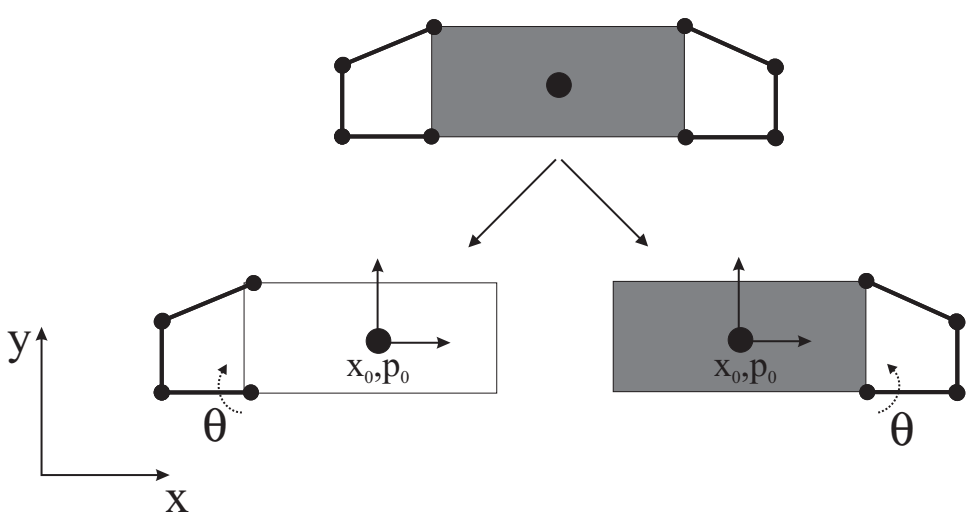

Fig. 1 Reduced coordinates for a SS-GMP

The reduced sub-model is described by the position $x_{0}$ and orientation $p_{0}$ of its moving reference frame and the relative mechanism motion with respect to this frame, denoted by a minimal set of coordinates $\theta_{i}$, as shown in Fig. 1 . In this work no linearizations are performed for the model reduction, but this could be added to the formulation in accordance to Naets [11].

Through a nonlinear transformation, the unreduced DOFs of a sub-system $x_{i}$ and their derivatives can be obtained from the reduced coordinates ${ }^{1}$ :

$$
\begin{aligned}
x= & x_{0}+R\left(p_{0}\right) \rho\left(\theta_{i}\right), \\
\dot{x}= & \dot{x}_{0}+R\left(p_{0}\right)_{, p_{0}} \dot{p_{0}} \rho\left(\theta_{i}\right)+R\left(p_{0}\right) \Psi^{x \theta_{i}} \dot{\theta}_{i}, \\
\ddot{x}= & \ddot{x}_{0}+2 R\left(p_{0}\right)_{, p_{0}} \dot{p_{0}} \Psi^{x \theta_{i}} \dot{\theta}_{i}+R\left(p_{0}\right)_{, p_{0}, p_{0}} \dot{p}_{0}^{2} \rho\left(\theta_{i}\right) \\
& +R\left(p_{0}\right) \Psi_{, \theta_{i}}^{x \theta_{i}} \dot{\theta}_{i}^{2}+R\left(p_{0}\right)_{, p_{0}} \ddot{p_{0}} \rho\left(\theta_{i}\right)+R\left(p_{0}\right) \Psi^{x \theta_{i}} \ddot{\theta}_{i} .
\end{aligned}
$$

Due to the use of the MAF, a rotation matrix $R\left(p_{0}\right)$ is required, which transforms the coordinates expressed with respect to the local frame to the global frame. In these equations, the nonlinear transformation function $\rho\left(\theta_{i}\right)$ is defined, which relates the unreduced coordinates with respect to the MAF to a minimal set of reduced coordinates $\theta_{i}$. The derivative of this nonlinear function is defined as:

$$
\rho_{, \theta_{i}}=\Psi^{x \theta_{i}}
$$

which is a set of projection modes which are also dependent on $\theta_{i}$. In the GMP formalism, these nonlinear functions are determined by sampling the configuration of the system over a predetermined grid of possible configurations. These configurations are stored and during simulation an Overhauser interpolation is used on this grid, which allows a continuous function between the sampling points up to the first derivative. The interpolation for a configuration $\theta_{i}$ in the interval $\left[\theta_{i}^{k}, \theta_{i}^{k+1}\right]$ for a matrix $A$ with derivatives $d A$ is then given by:

$$
\begin{aligned}
s= & \frac{\theta_{i}-\theta_{i}^{k}}{\theta_{i}^{k+1}-\theta_{i}^{k}}, \\
A= & \left(1-3 s^{2}+2 s^{3}\right) A^{k}+\left(3 s^{2}-2 s^{3}\right) A^{k+1} \\
& +\left(s-2 s^{2}+s^{3}\right) d A^{k}+\left(-s^{2}+s^{3}\right) d A^{k+1} .
\end{aligned}
$$

\footnotetext{
1 the derivative of a matrix $A$ with respect to a variable $b$ is denoted as $A_{, b}$
} 
This choice of interpolation function leads to an acceptable level of continuity at minimal cost.

With the degrees-of-freedom for each sub-model defined, the equations of motion for each model can be derived.

\subsection{Equations of motion for sub-models}

This section briefly reviews the equations of motion for a sub-model. It is important to notice that these equations are the same, irrespective of the original modeling approach. This is a major advantage because it implies that the proposed filtering formalism can be used to generate a KF for any original multibody modeling approach without the necessity to perform any additional derivations of the model to obtain it in a KF-eligible form.

This section also offers the exact derivatives of the different forces with respect to the reduced DOFs because these will be crucial for a proper operation of the extended Kalman filter as described in Sec. 3. In this section only the final equations are provided, for a full derivation of these equations the reader is referred to [11]. The forces which make up the equations of motion can be split into: inertial forces, internal forces and external forces.

Inertial forces. The inertial forces constitute the velocity dependent gyroscopic forces and the acceleration dependent forces.

An additional projection matrix $D$ is required in order to evaluate the inertial forces:

$$
D=\left[\begin{array}{cc}
R\left(p_{0}\right)^{T} & 0 \\
0 & I_{1+m^{\theta}}
\end{array}\right]
$$

This transformation matrix transforms the sub-system coordinates $q_{i}$ to the MAF, with $m^{\theta}$ the number of minimal mechanism coordinates. With this projection matrix the sub-system mass-matrix is:

$$
M_{i}=D^{T} M_{c}\left(\theta_{i}\right) D
$$

In this equation, $M_{c}\left(\theta_{i}\right)$ is the reduced mass-matrix with respect to the MAF, which is dependent on the configuration of the system $\theta_{i}$ with respect to the MAF. The reduced mass-matrix is obtained by projecting the unreduced mass-matrix as described by Naets [11], such that the online computations are independent of the unreduced number of DOFs. This matrix is also stored during the preprocessing and obtained through interpolation during the simulation. For the gyroscopic forces, the derivatives of the mass-matrix with respect to the DOFs $q_{i}$ are required:

$$
\begin{aligned}
M_{i, p_{0}} & =D_{, p 0}^{T} M_{c}\left(\theta_{i}\right) D+D^{T} M_{c}\left(\theta_{i}\right) D_{, p 0} \\
M_{i, \theta_{i}} & =D^{T} M_{c, \theta_{i}}\left(\theta_{i}\right) D
\end{aligned}
$$

Just as the local mass-matrix, also the derivative of the local reduced mass-matrix $M_{c, \theta_{i}}$ is stored and this allows the use of the cubic Overhauser interpolation for the mass-matrix. The gyroscopic forces on DOF $j$ can then be computed as:

$$
F_{g y r}^{j}=\sum_{k=1}^{m^{q}}\left(M_{i, k}^{j} \dot{q}_{i}^{k}\right) \dot{q}_{i}-\frac{1}{2} \dot{q}_{i}^{T} M_{i, j} \dot{q}_{i} .
$$


The required derivatives of the gyroscopic forces with respect to the position and velocity of $q_{i}^{l}$ are:

$$
F_{g y r, q_{i}^{l}}^{j}=\sum_{k=1}^{m^{q}}\left(M_{i, k, l}^{j} \dot{q}_{i}^{k}\right) \dot{q}_{i}-\frac{1}{2} \dot{q}_{i}^{T} M_{i, j, l} \dot{q}_{i},
$$

with

$$
\begin{aligned}
M_{i, p_{0}, p_{0}} & =D_{, p 0, p 0}^{T} M_{c}\left(\theta_{i}\right) D+D^{T} M_{c}\left(\theta_{i}\right) D_{, p 0, p 0}+2 D_{, p 0}^{T} M_{c}\left(\theta_{i}\right) D_{, p 0}, \\
M_{i, p_{0}, \theta_{i}} & =D_{, p 0}^{T} M_{c, \theta_{i}}\left(\theta_{i}\right) D+D^{T} M_{c, \theta_{i}}\left(\theta_{i}\right) D_{, p 0} \\
M_{i, \theta_{i}, p_{0}} & =D_{, p_{0}}^{T} M_{c, \theta_{i}}\left(\theta_{i}\right) D+D^{T} M_{c, \theta_{i}}\left(\theta_{i}\right) D_{, p_{0}} \\
M_{i, \theta_{i}, \theta_{i}} & =D^{T} M_{c, \theta_{i}, \theta_{i}}\left(\theta_{i}\right) D .
\end{aligned}
$$

and

$$
F_{g y r, \dot{q}_{i}^{l}}^{j}=\sum_{k=1}^{m^{q}}\left(M_{i, k}^{j l} \dot{q}_{i}^{k}\right)+M_{i, l}^{j} \dot{q}_{i}-\frac{1}{2}\left(\dot{q}_{i}^{T} M_{i, j}^{l}+M_{i, j}^{l} \dot{q}_{i}\right) .
$$

With these forces and derivatives, the inertial contributions to the equations of motion required for the Kalman filter can be evaluated.

Internal forces. Even though the current work focuses on rigid multibody systems, internal forces can still be present due to the presence of force elements (eg. springs) in the model. For the case where a force-element is acting on the MAF, these contributions are considered as external forces because these are not internal to the reduced model. Only the forceelements which are acting on the relative rigid DOFs $\theta_{i}$ are considered as internal forces. The reduced internal forces $F_{i n t}^{q_{i}}$ are also obtained by projecting the unreduced (nonlinear) internal forces $F_{i n t}^{x}$ onto the reduced DOFs, similar to the process described in [28]:

$$
\begin{aligned}
F_{i n t}^{x_{0}} & =0, \\
F_{i n t}^{p_{0}} & =0, \\
F_{\text {int }}^{\theta_{i}} & =\left(\Psi^{x \theta_{i}}\right)^{T} F_{\text {int }}^{x}\left(\rho\left(\theta_{i}\right)\right),
\end{aligned}
$$

with associated generalized stiffness-matrix $F_{i n t, q_{i}}^{q_{i}}$ fully zero, except for :

$$
F_{i n t, \theta_{i}}^{\theta_{i}}=\left(\Psi^{x \theta_{i}}\right)^{T} F_{i n t, x}^{x}\left(\rho\left(\theta_{i}\right)\right) \Psi^{x \theta_{i}}+\left(\Psi_{, \theta_{i}}^{x \theta_{i}}\right)^{T} F_{i n t}^{x}\left(\rho\left(\theta_{i}\right)\right) .
$$

These vectors and matrices are again stored and interpolated during simulation.

External forces. The external forces (these are generalized forces so this also applies to torques) are assumed to be defined to be acting upon the unreduced coordinates $x$. In order to get the effect on the reduced DOFs, they should be projected on the derivative of Eq. (2):

$$
F_{\text {ext }}^{q_{i}}=x_{, q_{i}}^{T} F_{\text {ext }}^{x} .
$$

In order to be able to evaluate the KF properly, also the derivative of these forces are required:

$$
F_{e x t, q_{i}}^{q_{i}}=x_{, q_{i}, q_{i}}^{T} F_{e x t}^{x}+x_{, q_{i}}^{T} F_{e x t, x}^{x} x_{, q_{i}}
$$


The necessary additional derivatives to evaluate this function can be extracted from Eq. (3)(4).

All force contributions, except for the acceleration dependent forces, can be consolidated into a generalized force vector $g_{i}$ for sub-model $i$ :

$$
g_{i}\left(q_{i}, \dot{q}_{i}, F_{\text {ext }}^{x}\right)=-F_{\text {int }}\left(q_{i}\right)-F_{\text {gyr }}\left(q_{i}, \dot{q}_{i}\right)-x_{, q_{i}}^{T} F_{\text {ext }}^{x},
$$

such that the equations of motion for a sub-model can be written as:

$$
M_{i} \ddot{q}_{i}=g_{i}\left(q_{i}, \dot{q}_{i}, F_{\text {ext }}^{x}\right) .
$$

\subsection{First order system equations of motion}

In order to evaluate the full system, a back-transformation eliminating the redundant DOFs for the MAFs of the different sub-models has to be performed. This can be obtained by applying a linear projection $S$ onto the coordinates of the $n$ sub-models, such that no additional constraints need to be added to solve the equations of motion:

$$
S q=\left[\begin{array}{c}
q_{1} \\
\ldots \\
q_{n}
\end{array}\right],
$$

with

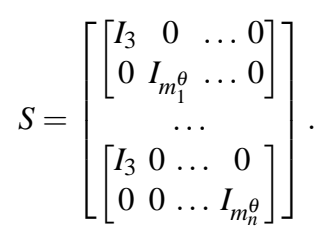

The full mass-matrix and force vector for the full model is composed from the matrices of the sub-models as:

$$
\begin{gathered}
M=S^{T}\left[\begin{array}{ccc}
M_{1} & \ldots & 0 \\
0 & \ldots & 0 \\
0 & \ldots & M_{n}
\end{array}\right] S, \\
g\left(q, \dot{q}, F_{\text {ext }}\right)=S^{T}\left[\begin{array}{c}
g_{1}\left(q_{1}, \dot{q}_{1}, F_{\text {ext }}^{x}\right) \\
\ldots \\
g_{n}\left(q_{n}, \dot{q}_{n}, F_{\text {ext }}^{x}\right)
\end{array}\right] .
\end{gathered}
$$

With this projection, the equations of motion for all the sub-systems can be combined into the non-redundant equations of motion for the full system:

$$
M \ddot{q}=g\left(q, \dot{q}, F_{\text {ext }}\right) .
$$

In previous works the equations of motion have always been presented in second-order form. The formulations for state-estimators are however usually derived for first-order systems (exceptions are eg. presented in the work by Hernandez [29]). Therefore, the equations of motion have to be written as:

$$
\dot{w}=f\left(w, F_{\text {ext }}\right),
$$


with

$$
w=\left[\begin{array}{c}
q \\
\dot{q}
\end{array}\right] .
$$

The nonlinear system equation $f$ for the case of these mechanical systems is:

$$
f\left(w, F_{\text {ext }}\right)=\left[\begin{array}{c}
\dot{q} \\
M^{-1} g\left(q, \dot{q}, F_{\text {ext }}\right)
\end{array}\right] .
$$

Furthermore, both the discretization scheme and the KF require the derivative matrix $F$ of this equation. The derivative of the generalized force-expression with respect to the minimal coordinates is:

$$
\begin{aligned}
& g_{, q}=S^{T}\left[\begin{array}{ccc}
g_{1, q_{1}}\left(q_{1}, \dot{q}_{1}, F_{\text {ext }}^{x}\right) & \ldots & 0 \\
\ldots & & \\
0 & \ldots & g_{n, q_{n}}\left(q_{n}, \dot{q}_{n}, F_{\text {ext }}^{x}\right)
\end{array}\right] S, \\
& g_{, \dot{q}}=S^{T}\left[\begin{array}{ccc}
g_{1, \dot{q}_{1}\left(q_{1}, \dot{q}_{1}, F_{\text {ext }}^{x}\right)} \ldots & 0 \\
\ldots & & \\
0 & \ldots & g_{n, \dot{q}_{n}}\left(q_{n}, \dot{q}_{n}, F_{\text {ext }}^{x}\right)
\end{array}\right] S .
\end{aligned}
$$

With these equations, $F$ becomes:

$$
\begin{aligned}
f\left(w, F_{\text {ext }}\right)_{, q} & =\left[\begin{array}{c}
0 \\
M^{-1} g\left(q, \dot{q}, F_{\text {ext }}\right)_{, q}-M^{-1} M_{, q} M^{-1} g\left(q, \dot{q}, F_{\text {ext }}\right)
\end{array}\right], \\
f\left(w, F_{\text {ext }}\right)_{, \dot{q}} & =\left[\begin{array}{c}
I_{n_{q}} \\
M^{-1} g\left(q, \dot{q}, F_{\text {ext }}\right)_{, \dot{q}}
\end{array}\right], \\
F & =\left[f\left(w, F_{\text {ext }}\right)_{, q} f\left(w, F_{\text {ext }}\right)_{, \dot{q}}\right] .
\end{aligned}
$$

With these equations, the system can be discretized and the extended Kalman filter can be evaluated. Even though the above derivation was made for the planar case (only one rotation parameter), these results can easily be generalized to the spatial case. In the following section these equations will be exploited to create a coupled state/input-estimator.

\section{Augmented discrete extended Kalman filter}

This work focuses on combined state and input filtering for mechanical systems. Regular Kalman filtering leads to bias-errors in the case of an unknown input, such that an adapted method has to be applied. In mechanical systems, the external inputs to the system are often unknown or very difficult to model. Moreover, it might be highly useful have an estimate of these unknown forces for many applications as well. In this work the augmented Kalman filter is used, in which the unknown forces $a$ are added as additional states to be estimated. This leads to the augmented state vector $w^{*}$ :

$$
w^{*}=\left[\begin{array}{l}
w \\
a
\end{array}\right]
$$

In order to estimate the unknown input forces, some form of model has to be provided for these variables. In this work a random walk model is used, with continuous time model equation:

$$
\dot{a}=r_{a} .
$$


In this equation $r_{a}$ is continuous time noise, which indicates that the rate of change is expected to be a random process. This approach has been applied with success for linear mechanical systems by Lourens [26] and higher order versions of this approach have been used by Ray $[17,18]$. The zeroth order model employed here allows good versatility for different input forces at a minimal computational load.

Many nonlinear Kalman filter formulations have been proposed over the years and in this work the discrete extended Kalman filter (DEKF) is employed. In order to use the continuous time equations provided in the previous section, the equations of motion need to be discretized. This discretization is discussed in Sec. 3.1. Subsequently, the algorithm for the A-DEKF approach is briefly reviewed in Sec. 3.2.

\subsection{Discretization of equations of motion}

The discrete extended Kalman filter is most suitable for real-time purposes because it is specifically developed for digital implementation and allows iteration-less integration. In order to be able to apply the DEKF to the continuous time equations of motion of multibody systems, the equations of motion need to be discretized.

With the inclusion of the augmented states for the unknown forces, the continuous time system equations are:

$$
\left[\begin{array}{c}
\dot{w} \\
\dot{a}
\end{array}\right]=\left[\begin{array}{c}
f\left(w, F_{\text {ext }}+S_{a} a\right) \\
0
\end{array}\right]+\left[\begin{array}{c}
0 \\
r_{a}
\end{array}\right] .
$$

In this equation $S_{a}$ is a projection matrix which projects the unknown forces to be estimated to the corresponding location in the full force-vector and $r_{a}$ is the noise-vector which determines the rate of change of the unknown forces. This equation can be summarized as:

$$
\dot{w}^{*}=f^{*}\left(w^{*}, F_{\text {ext }}\right)+r_{w^{*}} .
$$

This augmented function also has a new derivative matrix $F^{*}$ :

$$
\begin{aligned}
F^{*} & =f^{*}\left(w^{*}, F_{\text {ext }}\right)_{, w^{*}}, \\
& =\left[\begin{array}{cc}
F-M^{-1} x_{, q}^{T} S_{a} \\
0 & 0
\end{array}\right] .
\end{aligned}
$$

In order to perform the discretization many approaches exist. In this work a nonlinear exponential solver, more specifically the Exponentially Fitted Euler solver, is applied [27]. At a given time $t_{k}$ this method provides a solution for the states at $t_{k+1}$ :

$$
w_{k+1}^{*}=w_{k}^{*}+\int_{0}^{\Delta t} e^{F_{k}^{*} \eta} d \eta f^{*}\left(w_{k}^{*}, F_{\text {ext }}\right)
$$

Many different forms of the exponential solver for nonlinear systems exist and this approach is best suited for strongly nonlinear simulation, but it does require the computation of all derivatives at each timestep. The exact solution of the integral in this equation would require the computation of the inverse of $F_{k}^{*}$, but as is apparent from Eq. (45), this matrix is singular. To circumvent this problem, a Taylor approximation of the matrix-exponential is used instead:

$$
e^{F_{k}^{*} \eta} \approx I+\frac{F_{k}^{*} \eta}{1 !}+\frac{\left(F_{k}^{*}\right)^{2} \eta^{2}}{2 !}+\ldots+\frac{\left(F_{k}^{*}\right)^{n} \eta^{n}}{n !}+O\left(\eta^{n+1}\right)
$$


Approximating this exponential by a finite sum will limit the timestep for a stable solution, but this limitation will only be secondary to the limitation on the timestep due to the nonlinearity of the system. This polynomial function can be easily integrated such that the discretized equation of motion becomes (for an $n^{\text {th }}$ order approximation of the matrix exponential):

$$
\begin{aligned}
w_{k+1}^{*} & =f_{d}\left(w_{k}^{*}, F_{\text {ext }}\right), \\
& =w_{k}^{*}+\sum_{j=1}^{n}\left(\left(F_{k}^{*}\right)^{j-1} \frac{\Delta t^{j}}{j !}\right) f^{*}\left(w_{k}^{*}, F_{\text {ext }}\right) .
\end{aligned}
$$

Moreover, also the derivative $F_{d}$ of this function with respect to the states has to be computed for the Kalman filter:

$$
\begin{aligned}
F_{d} & =f_{d, w^{*}}, \\
& =I+\sum_{j=1}^{n}\left(\left(F_{k}^{*}\right)^{j-1} \frac{\Delta t^{j}}{j !}\right) F_{k}^{*}+\sum_{j=1}^{n-1}\left(\frac{\partial\left(F_{k}^{*}\right)^{j-1}}{\partial w^{*}} \frac{\Delta t^{j+1}}{j+1 !}\right) f^{*}\left(w_{k}^{*}, F_{e x t}\right) .
\end{aligned}
$$

In this equation the last contribution is neglected. This approximation will have limited effect on the accuracy of the filter. If the system is close to linear, the timestep can be selected relatively large but the derivative of $F^{*}$ will be negligible with respect to $F^{*}$, so this approximation is valid. If the system is strongly nonlinear, a relatively small timestep is required for accurate time-integration. This small timestep will lead to a negligible contribution of the last term with respect to the other terms. Moreover, computing the derivatives of $F^{*}$ would add considerable computational complexity and jeopardize real-time performance. With this approximation, the Jacobian matrix becomes:

$$
F_{d} \approx I+\sum_{j=1}^{n}\left(\left(F_{k}^{*}\right)^{j-1} \frac{\Delta t^{j}}{j !}\right) F_{k}^{*}
$$

Besides the regular equations of motion, also the discretized behavior of the expected noise on the model has to be considered. In this work, the noise on the continuous time multibody model is assumed to be zero and all noise is assumed on the unknown force, with covariance $R_{a}$. In this case the discretized noise vector for the augmented system $r_{w^{*}}$ becomes:

$$
r_{w^{*}} \approx F_{d}^{a} r_{a}
$$

and the covariance matrix for the discretized time states $R_{w^{*}}$ is:

$$
R_{w^{*}} \approx F_{d}^{a} R_{a}\left(F_{d}^{a}\right)^{T}
$$

In general $R_{a}$ is not exactly known and is used as a tuning parameter for the filter in order to get a satisfactory trade-off between the model and the measurements [26]. This is also the approach followed in this work. In the general case there is also uncertainty on the model, which can be taken into account by adjusting $r_{w^{*}}$ and $R_{w^{*}}$. This case is not investigated further in this work in order to focus on the problem of input-estimation. 


\subsection{A-DEKF algorithm}

The augmented discrete extended Kalman filter (A-DEKF) is proposed in this work to provide a coupled estimation of the states and inputs to a mechanical system.

The nonlinear discrete time system equations $f_{d}$, obtained by the algorithm described in Sec. 3.1, are:

$$
w_{k}^{*}=f_{d}\left(w_{k-1}^{*}, F_{e x t}\right)+r_{w^{*}}
$$

This equation is now in a form suitable for discrete extended Kalman filtering. The first part of this equation represents the nominal system equations and the second part represents the discretized process noise $r_{w^{*}}$ with covariance $R_{w^{*}}$ which expresses the uncertainty on the model.

The system equations of motion are complemented by the (nonlinear) measurement equations:

$$
y_{k}=h\left(w_{k}^{*}, F_{\text {ext }}\right)+r_{y} .
$$

In this equation $y_{k}$ contains the sensor measurements obtained by the (nonlinear) measurement equation $h$ with measurement noise $r_{y}$ with covariance $R_{y}$. In this work the measurement noise is assumed stationary and to be added linearly, but all theory can easily be extended to the case of nonlinear noise addition according to the EKF formalism.

With these system equations, the A-DEKF equations can be derived fully analogous to the equations for a regular DEKF. The defining property of the Kalman-filter is the fact that it aims to minimize the trace of the expected covariance $P_{k}$ of the error of the estimated states with respect to the real states [2]. For the above described set of equations the DEKFalgorithm for a timestep $k$ becomes [2]:

$$
\begin{aligned}
P_{k}^{-} & =F_{d} P_{k-1}^{+} F_{d}^{T}+R_{w^{*}}, \\
w_{k}^{*-} & =f_{d}\left(w_{k-1}^{*+}, F_{e x t}\right), \\
K_{k} & =P_{k}^{-} H^{T}\left(H P_{k}^{-} H^{T}+R_{y}\right)^{-1}, \\
w_{k}^{*+} & =w_{k}^{*-}+K_{k}\left(y_{k}-h\left(w_{k}^{*-}, F_{e x t}\right)\right), \\
P_{k}^{+} & =\left(I-K_{k} H\right) P_{k}^{-},
\end{aligned}
$$

with

$$
\begin{aligned}
F_{d} & =f_{d}\left(w_{k-1}^{*}, F_{\text {ext }}\right)_{, w^{*}}, \\
H & =h\left(w_{k}^{*}, F_{\text {ext }}\right)_{, w^{*}} .
\end{aligned}
$$

The calculation of the derivative of the measurement equations is usually rather straightforward and the derivative of the discretized system equations is discussed in the next section. In this DEKF approach, first an estimate of the states and error-covariance is computed in Eq. (57)-(58). Based on these estimates, the Kalman gain $K_{k}$ is computed which is used to correct the initial estimates based on the measurements in Eq. (60)-(61). It is interesting to notice that the estimation of the inputs is fully integrated in the regular Kalman filter, the changes are included in the model. 


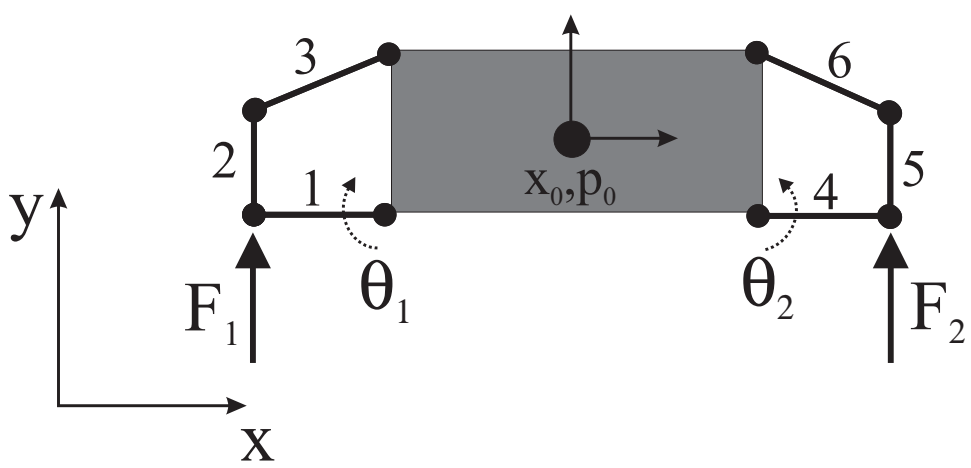

Fig. 2 Half-car modeled used for validation.

\section{Numerical validation}

In order to validate the proposed approach, a numerical validation of the A-DEKF with an SS-GMP model is performed. The validation is performed in Matlab and the proposed approach is compared to three different approaches to show consistently superior results. In order to validate the computational efficiency of the A-DEKF with an SS-GMP model, this method is also implemented in Fortran.

Firstly the model used is described. Next the different filtering methods are briefly discussed and finally the simulation results are shown.

\subsection{Model description}

In this work a half-car system, shown in Fig. 2, is used to validate the proposed coupled state/input-estimation approach. The system consists of a car-body and a four-bar suspension on the left and right side. The properties of each body are summarized in Table 1 .

Table 1 Properties of the different bodies of the half-car

\begin{tabular}{|c|cccc|}
\hline & mass $[\mathrm{kg}]$ & rot. iner. $\left[\mathrm{kgm}^{2}\right]$ & $\mathrm{b}[\mathrm{m}]$ & $\mathrm{h}[\mathrm{m}]$ \\
\hline car-body & 400 & 100 & 0.5 & 2 \\
beam 1 & 5 & 1 & 0.5 & $/$ \\
beam 2 & 3 & 0.7 & 0.3 & $/$ \\
beam 3 & 6 & 1.3 & 0.5385 & $/$ \\
beam 4 & 5 & 1 & 0.5 & $/$ \\
beam 5 & 3 & 0.7 & 0.3 & $/$ \\
beam 6 & 6 & 1.3 & 0.5385 & $/$ \\
\hline
\end{tabular}

The suspension is controlled by a spring at each side. These springs each have a constant stiffness of $40 \mathrm{kN} / \mathrm{m}$ and have a linear force-displacement behavior. No dampers are added in this example.

As external loading, gravity and vertical wheel forces are applied. For the estimation, these forces are split into two sets. The gravity is added as known to the model, as are the static wheel forces as response to the gravity. The variable part of the wheel forces is 
assumed to be unknown and has to be estimated. In this validation there is no real wheelroad interaction model and this effect is simply modeled by applying vertical forces to the wheel hubs.

Five measurements are performed to feed back to the filter. Two accelerometer measurements are performed on the horizontal and vertical position of the body. These accelerometers are attached to the car-body and thus provide accelerations in the frame attached to the body:

$$
y_{a c c}=\left[\begin{array}{c}
\ddot{x}_{0}^{\prime} \\
\ddot{y}_{0}^{\prime}
\end{array}\right]=R\left(p_{0}\right)^{T}\left[\begin{array}{c}
\ddot{x}_{0} \\
\ddot{y}_{0}
\end{array}\right] \text {. }
$$

For the models in the filters, these accelerations are evaluated through the equations of motion, leading to nonlinear measurement equations. Furthermore, three gyroscopic measurements are performed: one measurement on the angular velocity of the car body and two measurements for the relative angular velocities of the lower suspension arms. These measurements are chosen because they present a realistic option since they can be performed by low-cost MEMS sensors. For the noise on the measurements, normal white noise is assumed with realistic values for MEMS sensors. The covariance for the sensors is provided in Table 2.

Table 2 Sensor covariances

\begin{tabular}{|l|c|}
\hline acc. $x_{0}$ & $0.8 \mathrm{~m} / \mathrm{s}^{2}$ \\
acc. $y_{0}$ & $0.8 \mathrm{~m} / \mathrm{s}^{2}$ \\
gyr. $p_{0}$ & $0.2 \mathrm{rad} / \mathrm{s}$ \\
gyr. $\theta_{1}$ & $0.2 \mathrm{rad} / \mathrm{s}$ \\
gyr. $\theta_{2}$ & $0.2 \mathrm{rad} / \mathrm{s}$ \\
\hline
\end{tabular}

It is important to notice that these measurements do not lead to an observable system. Over time this will lead to a divergence of the estimated covariance and the Kalman filter will deteriorate. The divergence manifests itself as a drift on the position-level coordinates and forces and the rate is dependent on the magnitudes of the covariances. For shorter timespans as presented in this paper and in $[17,18,26]$ this is not an issue because the rate of drift is sufficiently low. For long term estimation purposes position level measurements (such as inclinometers, optical positioning systems, GPS) or stabilizing techniques [30] need to be added in order to obtain reliable results.

A sampling frequency of $1 \mathrm{kHz}$ is used in this work for the measurements and the timeintegration for the filters is run at the same frequency so there is a measurement for each filtering step.

Reference model. The reference model is expressed in Cartesian coordinates for the center of gravity for each body. The constraints on the multibody-system are taken into account through an R-projection approach [31]. The equations of motion are integrated with a generalized $\alpha$-solver [32] with timestep $1 \mathrm{~ms}$ and spectral radius $\rho_{\infty}=0.8$.

SS-GMP model. The SS-GMP reduced model consists of 2 sub-models, as shown in Fig. 1. The mass of the car-body is fully assigned to one of the two sub-models and the car-body is just a dummy body in the other sub-model. The MAF is attached at the center of gravity of the car body for each sub-model. For the parameterization of the relative mechanism motion $\theta$, the angle between the car-body and the lower-suspension arm is chosen in each model. 
With these choices each sub-model has four DOFs and the full model has five DOFs. The possible configurations span the range $\theta=[-0.4,0.63] \mathrm{rad}$ with a discretization step of $\Delta \theta=2 \mathrm{mrad}$. The storage of the reduced model requires $1.3 \mathrm{MB}$.

\subsection{Filters for comparison}

In order to create some frame of reference for the proposed A-DEKF, this method is compared to three other methods. In the following paragraphs each method is briefly discussed for this application. In each case the model covariances need to be tuned depending on the input-force. An input force with a larger derivative (eg. higher frequency) requires a larger force covariance.

Separate Kalman filtering with model inversion. The most straightforward approach to the problem of performing the state estimation and the input estimation of this system, would be to split up the problem into a separate state estimation and use this to compute the inputs through a system inversion, which is a simple linear problem for a given configuration in this case. Moreover it is also possible to neglect a system model altogether and use a full integrator approach, in which each measurement signal is filtered separately. This leads to a disturbance observer [25]. The continuous time state equations which allow the use of the acceleration measurements are:

$$
\left[\begin{array}{c}
\dot{q} \\
\ddot{q} \\
\dddot{q}
\end{array}\right]=\left[\begin{array}{lll}
0 & 1 & 0 \\
0 & 0 & 1 \\
0 & 0 & 0
\end{array}\right]\left[\begin{array}{c}
q \\
\dot{q} \\
\ddot{q}
\end{array}\right]+\left[\begin{array}{c}
0 \\
0 \\
r \ddot{q}
\end{array}\right] .
$$

This leads to linear system equations for the filter so a regular discrete Kalman filter (DKF) can be used to filter the states. In this case the covariance matrix for each separate signal has to be tuned for optimal performance. This leads to covariances for the accelerations of the different variables which are given in Table 3. It has to be noted that the acceleration

Table 3 State covariances for DKF estimator

\begin{tabular}{|c|c|}
\hline$R \dddot{x}_{0}$ & $1 e-3\left(\mathrm{~m} / \mathrm{s}^{3}\right)^{2}$ \\
$R \dddot{y}_{0}$ & $1 e-3\left(\mathrm{~m} / \mathrm{s}^{3}\right)^{2}$ \\
$R \dddot{p}_{0}$ & $1 e 1\left(\mathrm{rad} / \mathrm{s}^{3}\right)^{2}$ \\
$R \dddot{\theta}_{1}$ & $1 e 4\left(\mathrm{rad} / \mathrm{s}^{3}\right)^{2}$ \\
$R \dddot{\theta}_{2}$ & $1 e 4\left(\mathrm{rad} / \mathrm{s}^{3}\right)^{2}$ \\
\hline
\end{tabular}

measurements are performed in a vehicle attached frame, so the estimation of the angle of the body $p_{0}$ has to be performed first in order to allow for a projection of the acceleration measurements to obtain the acceleration in the global reference frame. This approach is referred to as the $D K F$ method in this paper.

A-DKF with linearized model. The second estimator which is considered, is the linear augmented discrete Kalman filter (A-DKF). In this approach the system equations are approximated by a linearization of the multibody equations around the initial configuration of the system. In this case the two unknown forces are also added as states according to the higher described random walk method. This approach is added in order to highlight the added value 
of using a multibody model instead of a linearized model, which is still common practice. In this case only two parameters need to be tuned, being the covariances for the two unknown forces. Since both forces can be assumed to have similar behavior, both are assigned the same expected covariance. After manual optimization the covariance for the unknown forces was set to $R_{a}=1 e 5(\mathrm{~N} / \mathrm{s})^{2}$. This approach is referred to as the $A-D K F$ method in this paper.

DEKF with model inversion. In this case a discrete extended Kalman filter (DEKF) is applied to the nonlinear SS-GMP model. For this estimator the states are not augmented with the unknown input forces and all forces are assumed known. The uncertainty to the model is located in the force equilibrium. The covariance matrix on the states is computed similar to the augmented case and the force covariance is fixed at $R_{a}=1 e 6(N)^{2}$. In this approach, the unknown forces are evaluated by applying the estimated states to the inverse model of the system, which again lead to a disturbance observer [25]. This approach is referred to as the $D E K F$ method in the remainder of this chapter.

$A-D E K F$. Finally the proposed method in this paper is applied. The augmented discrete extended Kalman filter is applied to the nonlinear SS-GMP model as described in the previous sections with the two unknown external forces as augmented states. The uncertainty concentrated in the rate of change of the unknown input-forces and their covariance after tuning is set at $R_{a}=1 e 6(\mathrm{~N} / \mathrm{s})^{2}$. This model is also implemented in Fortran in order to verify the real-time capability. This approach is referred to as the $A-D E K F$ method in this paper.

\subsection{Simulation results}

For the validation, a simulation of 0.4 seconds is performed. In this simulation a constant force, equal to half the reaction force for the gravitational force, augmented by a sinusoidal force is applied at each wheel. These input forces are shown in Fig. 3.

First of all, the tracking behavior for the measurements is compared. Fig. 4 shows the time history of the measurements and their filtered counterparts for the A-DEKF approach proposed in this work. The strength of the Kalman filtering approach is immediately apparent when considering the evolution of the $\ddot{x}_{0}$-measurement. Due to the noise of the accelerometer this signal is completely distorted but after the filtering it looks much better. All other considered filters provide rather good accuracy for the measured variables so they are not shown in this figure.

In order to get a more comprehensive view of the performance of the different filters, Fig. 5 shows the average variance of the five filtered states with respect to the exact states and their derivatives ${ }^{2}$ :

$$
\sigma\left(q^{i}\right)^{2}=\sum_{k=1}^{n_{t}} \frac{\left(q_{\text {est }}^{i}-q_{\text {truth }}^{i}\right)^{2}}{n_{t}} .
$$

Fig. 5 indicates that the A-DEKF is able to produce overall more reliable results than the three other filters. The DKF method provides rather inconsistent results over the different variables since each is handled by a separate filter. It is also apparent that the A-DKF approach is not able to properly track the behavior of $x_{0}$ because this motion is caused by nonlinear couplings in the model which are not present in the linearized model. The DEKF

\footnotetext{
2 These variances are averaged over 50 simulation-runs with random noise generated by randn in MATLAB.
} 

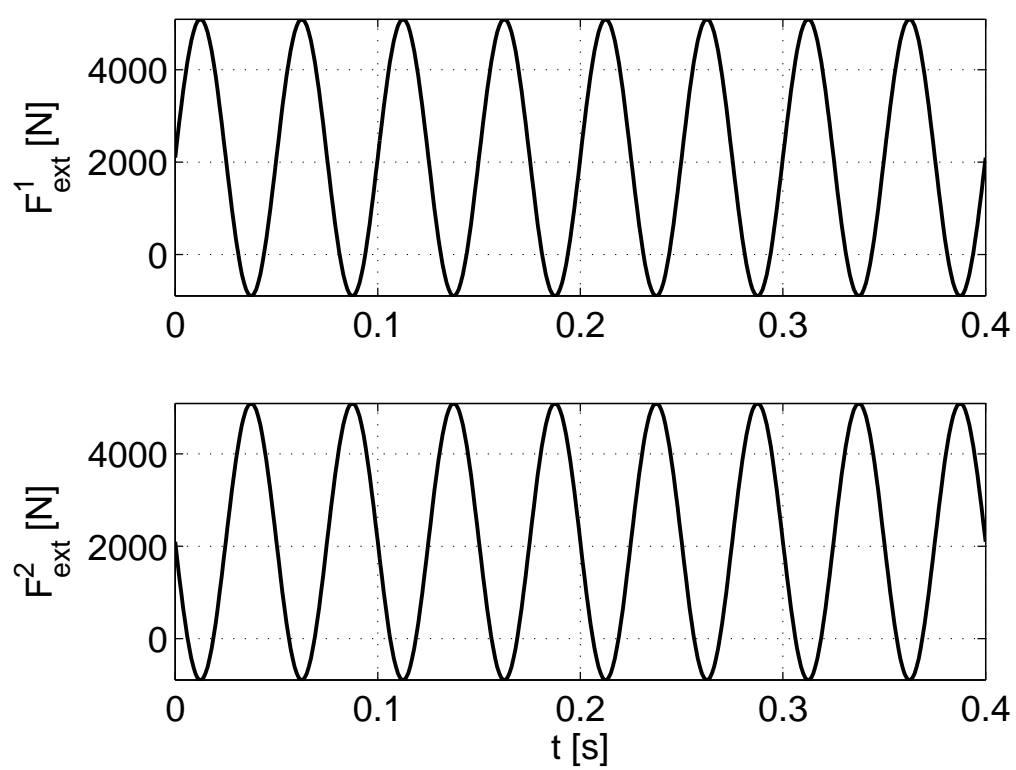

Fig. 3 Applied input forces to reference simulation (truth-model).

approach also clearly leads to bigger errors than the A-DEKF approach because the unknown input typically leads to biases in the results of the filter.

Furthermore also the behavior of the estimates of the variation on the input forces has to be considered. Fig. 6 shows the course of the two estimated forces and Fig. 7 shows the averaged covariances between the exact forces and the estimates. Fig. 6-7 further enforce the conclusion that the A-DEKF approach is able to deliver accurate results. All three model based approaches (A-DKF, DEKF and A-DEKF) however seem to provide relatively accurate results whereas the DKF method clearly provide inferior performance. This is mainly due to the poor tracking of $\theta_{1}$ and $\theta_{2}$ by the DKF approach and these variables are crucial for the spring-forces. The estimated forces show a slight lag with respect to the real force. This is logical since the resulting estimate will be somewhere between the actual force and the force predicted by the random walk model which predicts a constant force. Other force models might provide better results for this case but could compromise in versatility for other force shapes.

The above results clearly demonstrate that the A-DEKF approach delivers consistently superior results to the other approaches. Furthermore, the FORTRAN simulation of the ADEKF estimator with the SS-GMP model only takes 0.05 seconds for 0.4 seconds of simulation. Real-time performance can easily be obtained for the considered system with the proposed A-DEKF algorithm with an SS-GMP model ${ }^{3}$.

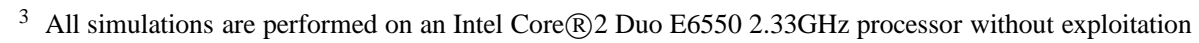
of multi-threading 

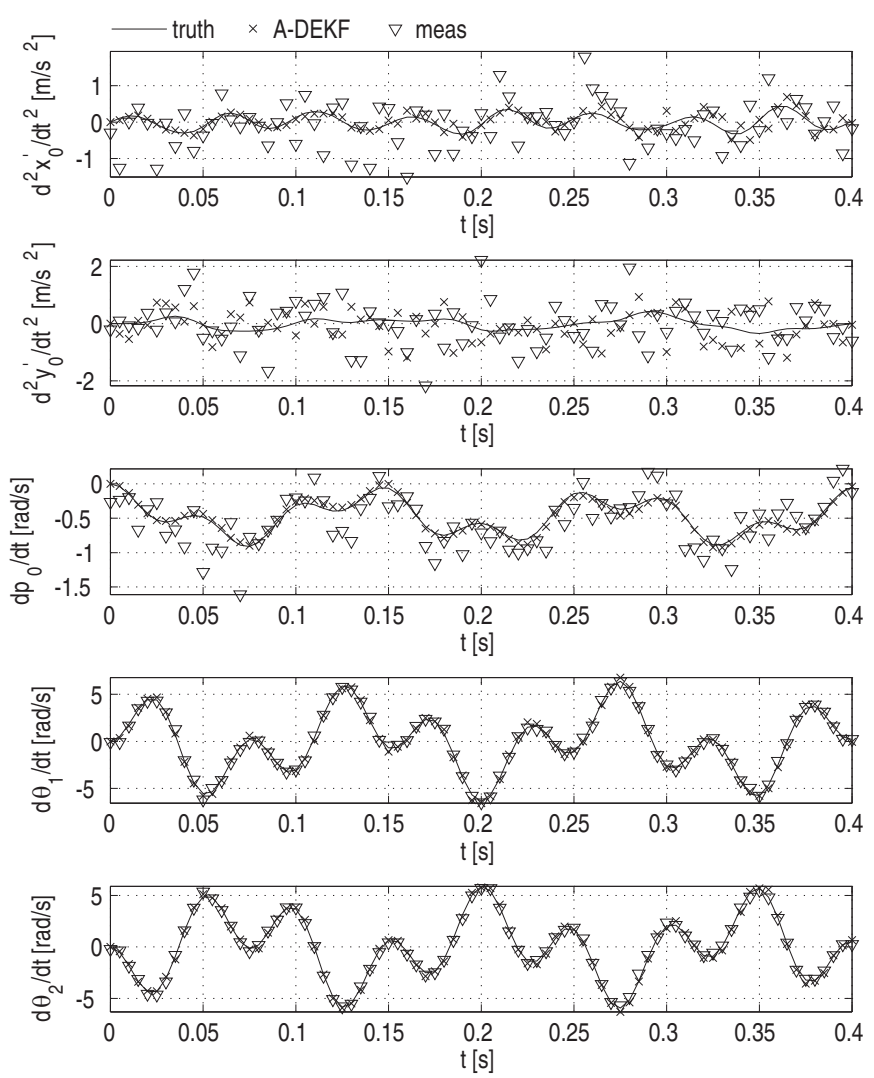

Fig. 4 Tracking of measured variables.

\section{Conclusion}

In this paper a methodology is proposed to use Sub-System Global Modal Parameterization (SS-GMP) reduced multibody models in an augmented discrete extended Kalman filter (A-DEKF) to generate a general formalism for online coupled state/input estimation for mechanical systems. The use of the SS-GMP approach allows generating real-time capable models from high fidelity multibody models of a mechanical system. Special attention is paid to the derivation of the nonlinear Jacobians of the equations of motion which are required for an accurate evaluation of the Kalman filter.

In many mechanical applications it is essential to provide an estimation of the external input forces since these might be very difficult if not impossible to determine in advance. In order to allow simultaneous state and input estimation, an augmented Kalman approach is adopted in which the unknown forces are added as additional states to be estimated. A discrete version of the filter is employed because this allows for a more efficient implementation. An additional novelty is the use of an exponential discretization scheme for the nonlinear SS-GMP equations of motion in order to match the model to the filter. Through a numerical validation the accuracy of the proposed A-DEKF filter with SS-GMP model is 

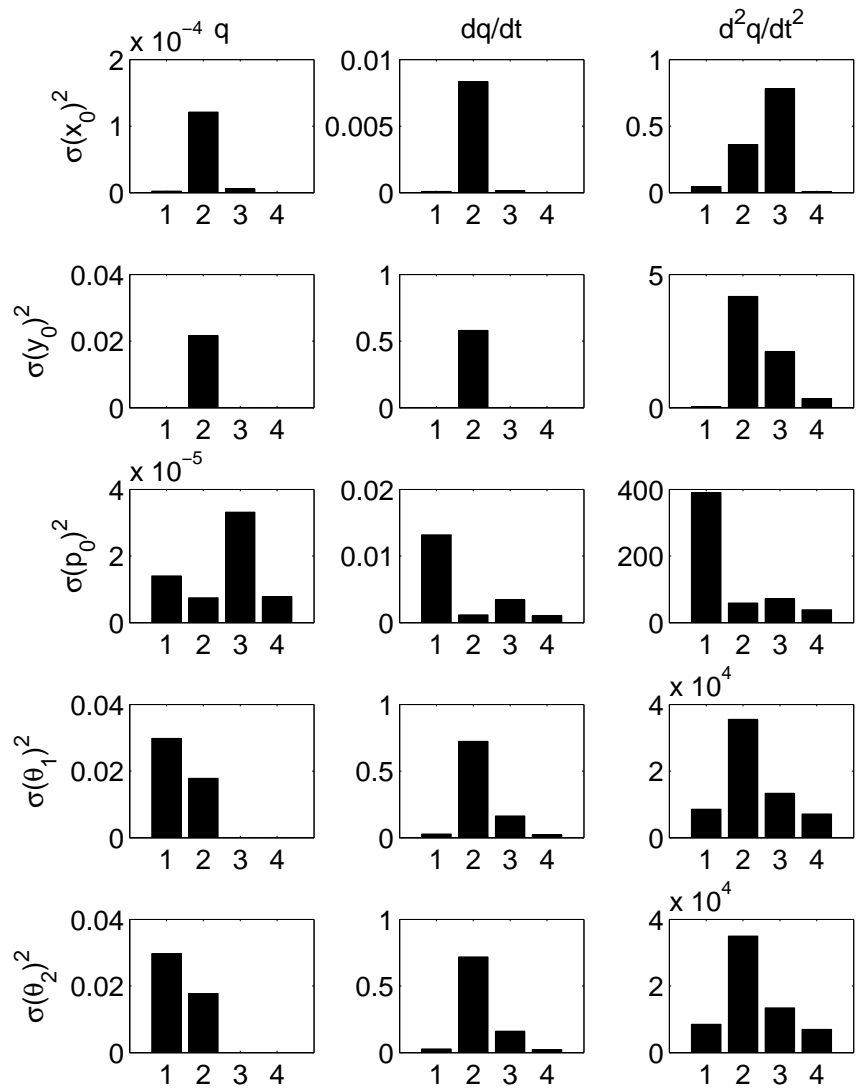

$1=$ DKF w. inv. mod, $2=A-D K F, 3=$ DEKF w. inv. mod, $4=A-D E K F$

Fig. 5 Average covariance of error on measured variables.

shown and the Fortran implementation of this formalism is able to run faster than real-time on a standard PC.

It has to be remarked that the proposed approach is not robust with respect to wrong initial conditions because this state cannot be observed with the current sensor setup. This non-observability will also eventually lead to divergence of the Kalman-filter. In order to remedy these issues position level measurements, such as a GPS signal for the body, have to be added. Future research will focus on these observability issues and the effects of different (biased) noise sources such as engine noise and aerodynamic effects.

Acknowledgements The research of Frank Naets is funded by a Ph.D grant of the Institute for the Promotion of Innovation through Science and Technology in Flanders (IWT-Vlaanderen) and his research stay at LIMUDC was funded by a grant from the Fund of Scientific Research (FWO). 

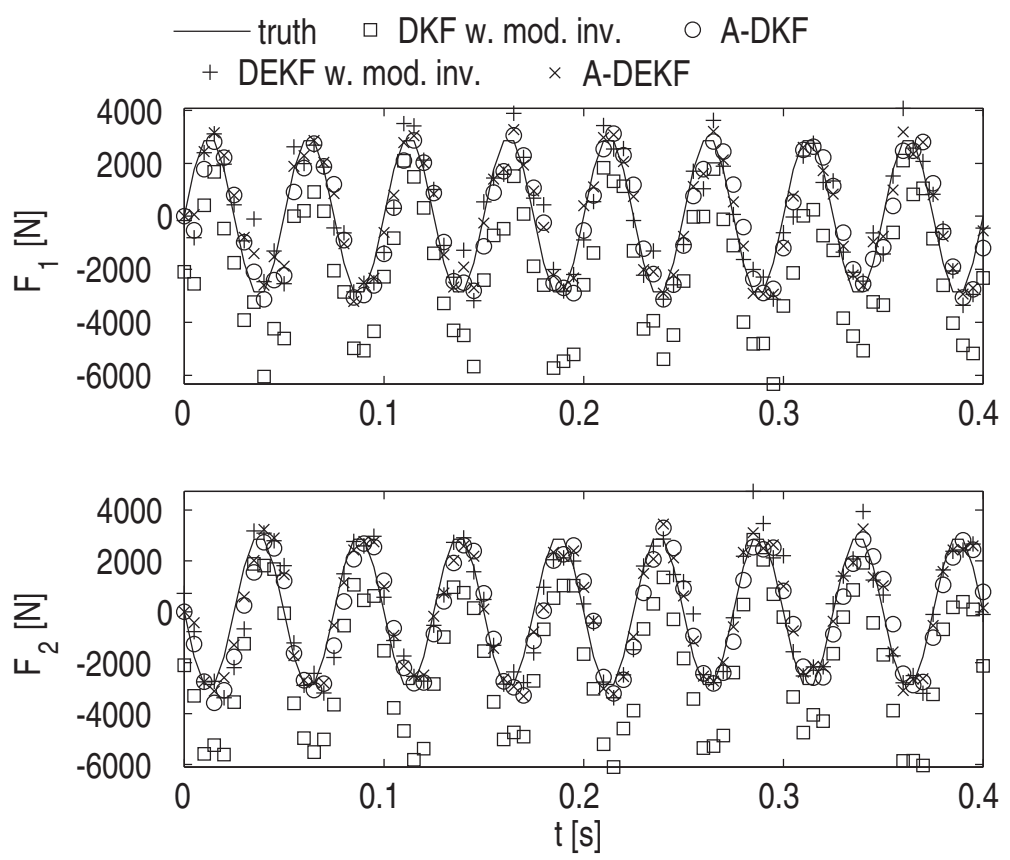

Fig. 6 Estimated input forces.
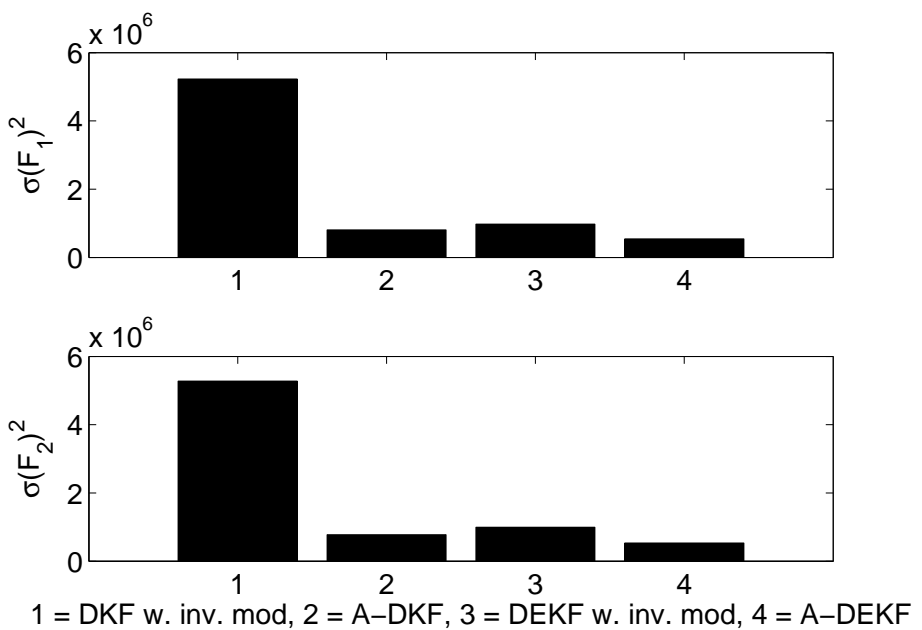

Fig. 7 Averaged covariance of error on input force estimates.

\section{References}

1. R.E. Kalman. A new approach to linear filtering and prediction problems. Journal of Basic Engineering, 82(1):35-45, 1960.

2. D. Simon. Optimal State Estimation: Kalman, $H_{\infty}$ and nonlinear approaches. J. Wiley and Sons, New York, 2006. 
3. P.M. Siegrist and P.R. McAree. Tyre-force estimation by kalman inverse filtering: applications to offhighway mining trucks. Vehicle System Dynamics: International Journal of Vehicle Mechanics and Mobility, 44(12):921-937, 2006.

4. J. Daklallah, S. Glaser, S. Mammar, and Y. Sebsadji. Tire-road forces estimation using extended kalman filter and sideslip angle evaluation. In Proceedings of the 2008 American Control Conference, 11-13 June, 2008.

5. H.F. Grip, L. Imsland, T.A. Johansen, T.I. Fossen, J.C. Kalkkuhl, and A. Suissa. Nonlinear vehicle side-slip estimation with friction adaptation. Automatica, 44(3):611-622, 2008.

6. T. Wasfy and A. Noor. Computational strategies for flexible multibody systems. Applied Mechanics Reviews, 56(6):553-613, 2002.

7. J. Cuadrado, D. Dopico, A. Barreiro, and E. Delgado. Real-time state observers based on multibody models and the extended kalman filter. Journal of Mechanical Science and Technology, 23(4):894-900, 2009.

8. J. Cuadrado, D. Dopico, J.A. Perez, and R. Pastorino. Automotive observers based on multibody models and the extended kalman filter. Multibody System Dynamics, 27(1):3-19, 2012.

9. O. Brüls, P. Duysinx, and J-C. Golinval. A model reduction method for the control of rigid mechanisms. Multibody System Dynamics, 15(3):213-227, 2006.

10. O. Brüls, P. Duysinx, and J-C. Golinval. The global modal parameterization for nonlinear model-order reduction in flexible multibody dynamics. Int. J. Numer. Meth. Engng, 69(5):948-977, 2007.

11. F. Naets, G.H.K. Heirman, and W. Desmet. Sub-system global modal parameterization for efficient simulation of flexible multibody systems. Int. J. Numer. Meth. Engng., 89(10):1227-1248, 2011.

12. E. Blanchard, A. Sandu, and C. Sandu. Parameter estimation for mechanical systems via an explicit representation of uncertainty. Engineering Computations. Int. J. for Computer-Aided Engineering and Software, 26(5):541-569, 2009.

13. E. Blanchard, A. Sandu, and C. Sandu. A polynomial chaos-based kalman filter approach for parameter estimation of mechanical systems. ASME J. of Dynamic Systems Measurement and Control, Special Issue on Physical System Modeling, 132(6), 2010.

14. E. Blanchard, A. Sandu, and C. Sandu. Polynomial chaos-based parameter estimation methods applied to vehicle system. Proc. of the Institution of Mechanical Engineers (IMechE) Part K: J. of Multi-Body Dynamics, 224(K1):59-81, 2010.

15. P.K. Kitanidis. Unbiased minimum-variance linear state estimation. Automatica, 23(6):775-778, 1987.

16. M Darouach and M. Zasadzinski. Unbiased minimum variance estimation for systems with unknown exogenous inputs. Automatica, 33(4):717-719, 1997.

17. L.R. Ray. Nonlinear state and tire force estimation for advanced vehicle control. IEEE Transactions on Control Systems Technology, 3(1):117-124, 1995.

18. L.R. Ray. Nonlinear tire force estimation and road friction identification: simulation and experiments. Automatica, 33(10):1819-1833, 1997.

19. J. Ishikawa and M. Tomizuka. Pivot friction compensation using an accelerometer and a disturbance observer for hard disk drives. IEEE Transactions on Mechatronics, 3(3):194-201, 1998.

20. K. Szabat and T. Orlowska-Kowalska. Performance improvement of industrial drives with mechanical elasticity using nonlinear adaptive kalman filter. IEEE Transactions on industrial electronics, 55(3):1075-1084, 2008.

21. C. Mitsantisuk, S. Katsura, and K. Ohishi. Kalman-filter-based sensor integration of variable power assist control based on human stiffness estimation. IEEE Transactions on industrial electronics, 56(10):38973905, 2009

22. G.H. Hostetter and J.S. Meditch. On the generalization of observers to systems with unmeasurable, unknown inputs. Automatica, 9(6):721-724, 1973.

23. C.-K. Ma and C.-C. Ho. An inverse method for the estimation of input forces acting on non-linear structural systems. Journal of Sound and Vibration, 275(3-5):953-971, 2001.

24. S. Gillijns and B. De Moor. Unbiased minimum-variance input and state estimation for linear discretetime systems with direct feedthrough. Automatica, 43(5):934-937, 2007.

25. A. Radke and Z. Gao. A survey of state and disturbance observers for practitioners. In Proceedings of the 2006 American Control Conference, Minneapolis, Minnesota, USA, 14-16 June, 2006.

26. E. Lourens, E. Reynders, G. De Roeck, G. Degrande, and G. Lombaert. An augmented kalman filter for force identification in structural dynamics. Mechanical Systems and Signal Processing, 27:446-460, 2012.

27. J. Ros, R. Yoldi, A. Plaza, and J. Angeles. Exponential integration schemes in multibody dynamics. In Proceedings of the second joint international conference on Multibody System Dynamics, Stuttgart, Germany, May 29-June 12012.

28. F. Naets and W. Desmet. Super-element global modal parameterization for efficient inclusion of highly nonlinear components in multibody simulation. Multibody Syst. Dyn., doi:10.1007/s11044-013-9342-2, 2013. 
29. E.M. Hernandez. Optimal model-based state estimation in mechanical and structural systems. Structural control and health monitoring doi: 10.1002/stc.513, 2011.

30. F. Naets, J. Cuadrado, and W. Desmet. Stable force identification in structural dynamics using kalman filtering and dummy-measurements. Mechanical Systems and Signal Processing (SUBMITTED).

31. J. Garcia de Jalon and E. Bayo. Kinematic and dynamic siimulation of multibody systems. Springer, New York, 1994.

32. O. Brüls and M. Arnold. The generalized-alpha scheme as a linear multistep integrator: Towards a general mechatronic simulator. ASME Journal of Computational and Nonlinear Dynamics, 3(4), 2008. 